\title{
Analyse des déterminants de l'adoption de la Régénération Naturelle Assistée (RNA) dans la zone soudano-sahélienne : cas des cercles de Diéma et Kolokani au Mali
}

\author{
Mahamadou DIABY 1*, Yenizié KONE², Kalifa TRAORE ${ }^{1}$, Alpha Seydou MAIGA ${ }^{1}$ et \\ Anewin Moïse TOGO $^{3}$
}

\author{
${ }^{1}$ Institut d'Economie Rurale (IER), B.P. 258, Bamako, Mali. \\ ${ }_{2}^{2}$ Projet de Recherche sur les Politiques de Sécurité Alimentaire au Mali (PREPOSAM), B.P. 2906, Bamako, \\ Mali. \\ ${ }^{3}$ ONG World Vision Mali, B.P. 2347, Bamako, Mali. \\ *Auteur correspondant ; E-mail : mahamadou12000@yahoo.fr, Tél : +22366246038
}

\section{RESUME}

$\mathrm{Au} \mathrm{Mali,} \mathrm{les} \mathrm{systèmes} \mathrm{de} \mathrm{production} \mathrm{agricole} \mathrm{sont} \mathrm{fortement} \mathrm{tributaires} \mathrm{des} \mathrm{précipitations,} \mathrm{ce} \mathrm{qui}$ explique l'impact direct de la variabilité du climat sur la sécurité alimentaire et les moyens de subsistance des ménages agricoles. La Régénération Naturelle Assistée (RNA) est l'une des stratégies d'adaptation qui permet d'accroître la résilience des exploitations agricoles face aux aléas climatiques. Cette étude vise à analyser les déterminants de l'adoption de la RNA dans les cercles de Diéma et de Kolokani, au Mali. Un échantillonnage aléatoire a été utilisé pour enquêter 594 exploitations agricoles dont 363 adoptants la RNA et 231 non-adoptants. Les résultats de l'estimation du modèle Logit montrent que la formation en RNA, la cueillette, l'utilisation des fertilisants, la perception sur la fertilité des champs et les revenus entrainent une augmentation de la probabilité d'adoption de la RNA. Par contre l'âge, l'accès au crédit, la possession du bétail et la perception sur le choc climatique ont une influence négative sur l'adoption de la RNA. Les résultats de l'estimation du modèle Logit montrent que la formation en RNA, la cueillette, l'utilisation des fertilisants, la perception sur la fertilité des champs et les revenus entrainent une augmentation de la probabilité d'adoption de la RNA. Les analyses plus minutieuses seront réalisées avant, en cours et après chaque étape d'adoption de la RNA afin de déterminer les tendances spatio-temporelles de la RNA. L'extension de la recherche sur la RNA dans d'autres zones agroécologiques contribuera sans doute à la détermination des impacts de la RNA sur le bien-être des communautés rurales.

(C) 2020 International Formulae Group. All rights reserved.

Mots clés: enquête, adoptants, non adoptants, exploitations agricoles ; modèle Logit.

\section{Analysis of the determinants of Farmer Managed Natural Regeneration (FMNR) adoption in Sudano-Sahelian zone: case of Diema and Kolokani district, in Mali}

\begin{abstract}
In Mali, agricultural production systems are highly dependent on rainfall, which explains the direct impact of climate variability on food security and livelihoods of agricultural households. Farmer Managed Natural Regeneration (FMNR) is one of the adequate strategies that may increase the resilience of agricultural households facing to climate constraints. This study aims to analyze the determinants of FMNR adoption in the areas of Diema and Kolokani, in Mali. Random sampling was used to investigate 594 agricultural households,
\end{abstract}


which including 363 adopters of FMNR and 231 non-adopters. Results estimation from the Logit model show that FMNR training, harvesting, fertilizer utilization, perceptions of field fertility and income increase the probability of FMNR adoption. On the other hand, age, access to credit, possession of livestock and perception on the climate shock have a negative influence on FMNR adoption. The results of the logit model estimation show that training on FMNR, harvesting, fertilizer use, field fertility perception and income increase the probability of FMNR adoption. More detailed analyses will be conducted before, during and after each phase of FMNR adoption to determine the spatial-temporal trends of FMNR. The extension of FMNR research to other agro-ecological areas will undoubtedly contribute to the determination of FMNR impacts on rural communities' well-being.

(C) 2020 International Formulae Group. All rights reserved.

Keywords: survey, adopters, non-adopters, farms, logit model.

\section{INTRODUCTION}

L'agriculture pluviale est la principale activité de subsistance des populations de la zone aride d'Afrique de l'Ouest. Cette agriculture est sujette à des risques élevés de production agricole plongeant régulièrement les communautés dans des crises d'insécurité alimentaire (OCHA, 2014).

Dans ces conditions, un certain nombre de stratégies d'adaptation techniques, économiques et sociales ont été mises au point (Mortimore et Adams, 2001). Ces stratégies portent essentiellement sur l'intégration de l'élevage dans les activités agricoles, la diversification des moyens de subsistance et l'émigration vers les villes. Mais, de sérieux problèmes (dont le changement climatique) existent quant à la mise en œuvre de ces stratégies, puisque la majorité des ménages ruraux sont pauvres avec un faible niveau de résilience.

En effet, la quatrième évaluation du Groupe d'experts intergouvernemental sur l'évolution du climat (GIEC) estime que la durée de la période de croissance des plantes dans les zones arides et semi-arides de l'Afrique de l'Ouest sera réduite de $20 \%$ d'ici 2050 (Boko et al., 2007). En outre, la dégradation des terres réduit la résilience des populations dans les systèmes de production pluviaux (Serme et al., 2015 ; Bayala et al., 2014). Une étude récente (FAO et ITPS, 2015) a montré que la dégradation des sols est en augmentation dans la plupart des pays
d'Afrique subsaharienne avec plus de $20 \%$ des terres déjà dégradées et affectant plus de $65 \%$ de la population. Cette situation, combinée avec le changement climatique, nécessitent la génération et l'adoption rapide de pratiques de résilience pour réduire la vulnérabilité des populations surtout de celles qui dépendent de l'agriculture pluviale.

Plusieurs techniques ont été mises en place par la recherche et la vulgarisation dans les pays de la région pour répondre à cette situation notamment: (i) les techniques de conservation du sol et de l'eau (zaï, demi-lune, cordon pierreux, etc.) ; (ii) les techniques de gestion intégrée des cultures (GIC) et (iii) les techniques de gestion intégrée de la fertilité du sol (GIFS).

La gestion intégrée de la fertilité du sol inclue les techniques d'agroforesterie comme la régénération naturelle assistée (RNA). La RNA est une pratique séculaire consistant à épargner et à entretenir dans la parcelle de culture, les régénérations naturelles spontanées à des densités désirées. Les jeunes plants d'arbres régénérés font l'objet de matérialisation (peinture, piquet) pour éviter leur prélèvement lors de travaux des champs. La RNA est possible partout où il $\mathrm{y}$ a des souches d'arbres vivants avec la possibilité de ré-germination ou de graines dans le sol qui vont germer. Des études récentes ont montré que la régénération naturelle assistée est une approche efficace pour lutter contre la dégradation des terres et accroître la résilience, 
la sécurité alimentaire et les revenus dans les zones arides (Bayala et al., 2014). Rosenstock et al. (2015) ont trouvé que la RNA permet d'augmenter durablement la productivité agricole, de renforcer la résilience des systèmes de production au changement climatique et de réduire les gaz à effet de serre provenant de l'agriculture. L'efficacité de la RNA dans la résilience des agriculteurs est renforcée s'ils participent aux marchés et si les moyens de subsistance sont diversifiés et si les femmes sont autonomes (FAO, 2016). Au Mali, plusieurs ONG évoluant en milieu rural ont des programmes de régénération naturelle assistée et sont appuyées en cela par des partenaires techniques et financiers. A cet égard, des résultats encourageants ont été obtenus par l'ONG Sahel ECO qui a réalisé dans la région de Mopti, 6457 ha de régénération naturelle assistée sur la base d'une densité moyenne de 156 pieds par ha (AEDD, 2017). Dans les régions de Kayes et de Koulikoro (Mali), quarante un mille trois cent quatorze (41 314) paysans ont adopté la RNA dans les cercles de Diéma et de Kolokani. Plus de 26000 hectares couverts par la RNA dans lesdites localités. La régénération naturelle assistée possède plusieurs avantages écosystémiques, améliore la disponibilité du bois, du fourrage, etc.

Malgré les nombreux attributs positifs de la RNA, son adoption par les producteurs reste très faible. Ce constat a été fait par Nkonya et al. (2016) dans une étude récente sur les différentes pratiques de gestion de la fertilité des sols qui conclue qu'une relation inverse existe entre leur rentabilité et leur adoption.

Le présent article vise à identifier les facteurs déterminants de l'adoption de la RNA dans la zone soudano-sahélienne au Mali.

\section{MATERIEL ET METHODES}

\section{Sites d'étude}

L'étude a été conduite dans deux sites situés à cheval entre la zone sahélienne et soudanienne, les cercles de Diéma et Kolokani au Mali (Figure 1). Le cercle de Diéma est à cheval entre les deux zones agro-climatiques tandis que Kolokani est localisé dans la zone soudano-sahélienne.

\section{Caractérisation de la zone sahélienne}

Au Mali, le sahel couvre $320000 \mathrm{~km}^{2}$, environ $26 \%$ du territoire national. Le Sahel correspond à des écosystèmes arides et semiarides, avec une pluviométrie moyenne annuelle variant entre 200 et $600 \mathrm{~mm}$. La zone sahélienne comprend cinq régions naturelles : Gourma, Plateau de Bandiagara-Hombori, Gondo-Mondoro, Hodh et Guidimagha. La végétation est composée de prairies aquatiques à graminées vivaces et steppes arbustives. Les arbres sont principalement des épineux du genre Acacia, accompagnés par endroits des espèces des genres Combretum, Boscia, etc.

\section{Caractérisation de la zone soudanienne}

La zone soudanienne couvre environ $17 \%$ du territoire national. La pluviométrie, répartie sur 5 mois, varie de $600 \mathrm{~mm} / \mathrm{an}$ au Nord à plus de $1200 \mathrm{~mm} / \mathrm{an}$ au Sud. La végétation est composée de savanes arborées et arbustives avec un couvert végétal plus ou moins dense et varié. On y rencontre des essences dites de valeurs clairsemées comme Vilellaria paradoxa (Karité), Parkia biglobosa (néré), Tamarindus indica (tamarinier), Adansonia digitata (baobab) et Khaya senegalensis (caïlcédrat) auxquelles s'ajoutent les Ficus sp, Lannea $s p$ au sud, les Combretacées, Bombax costatum et Sterculia setigera en remontant vers le Nord.

\section{La collecte des données}

Au total, 38 enquêteurs ont été retenus et repartis par binômes en deux équipes pour la collecte des données. Les données ont été collectées sur des tablettes auprès de 594 exploitations agricoles répartis entre les deux strates et selon leur poids. 


\section{Traitement et analyse des données}

Dans la littérature, les modèles linéaires et non linéaires (Logit, Probit, Tobit) sont souvent utilisés pour analyser les déterminants de l'adoption des technologies agricoles. Cependant parmi tous ces modèles, le Logit reste le plus utilisé en raison de sa simplicité et les avantages qu'il offre à l'interprétation des données.

Dans le cadre de notre étude, il s'agit d'expliquer les caractéristiques de l'adoption et la non adoption de la RNA par les exploitations agricoles. Pour cela nous avons opté pour le modèle de la régression logistique pour analyser les déterminants de l'adoption de la RNA dans la zone soudano-sahélienne au Mali. On considère un échantillon de $\mathrm{n}$ individus d'indices $\mathrm{i}=1$ à $\mathrm{n}$. Pour chaque individu et l'on pose : $\mathrm{Yi}=1$ (si la RNA est adoptée) et 0 (si la RNA n'est pas adoptée). Ce choix permet de définir la probabilité d'adoption comme espérance de la variable $Y$ puisque :

$\mathrm{E}[\mathrm{Yi}]=\operatorname{Pr}(\mathrm{Yi}=1) \times 1+\operatorname{Pr}(\mathrm{Yi}=0) \times 0=\operatorname{Pr}(\mathrm{Yi}=$ 1). L'espérance de Yi donne donc la probabilité que les exploitations adoptent la technologie. L'objectif de ce modèle consiste alors à expliquer l'adoption de la RNA en fonction de $\mathrm{K}$ caractéristiques observées (Xi1,..,XiK) pour un individu i de l'échantillon, par exemple l'âge du chef d'exploitation, le niveau d'éducation, l'accès au crédit.

$(\mathrm{Y})=\mathrm{X} \beta+\varepsilon, Y=\left\{\begin{array}{l}0 \text { si } Y^{*} \leq 0 \\ 1 \text { si } Y^{*} \geq 1\end{array}\right.$

$(\mathrm{Yi}=1)=\mathrm{X} \beta+\varepsilon$

Où $\mathrm{Y}^{*}$ est une variable sous-jacente ou variable latente,

$\mathrm{X}$ est un vecteur des covariantes qui détermine les caractéristiques de l'adoption de la RNA et $\beta$ est un vecteur des coefficients associés.

$\varepsilon$ représente l'erreur logistique de la distribution.

Les logiciels STATA et SPSS ont été utilisé respectivement pour les analyses économétriques et descriptives.

\section{Choix des variables du modèle}

Les études ont montré que plusieurs facteurs sont susceptibles d'influencer l'adoption de la régénération naturelle assistée par les exploitations agricoles. La variable dépendante du modèle empirique est l'adoption de la RNA. C'est une variable qualitative dichotomique qui prend la valeur 1 si l'exploitation agricole a adopté ladite technologie et 0 si non. Les variables explicatives du modèle sont au nombre de quinze. Il s'agit :

Genre du chef d'exploitation : Le genre $\mathrm{du}$ chef d'exploitation est une variable binaire qui prend la valeur 1 si le producteur est un homme et 0 s'il est une femme. Traditionnellement, en milieu rural la plupart des chefs d'exploitation sont des hommes. On peut supposer que le chef d'exploitation a un rôle important dans l'adoption d'une technologie.

Age du chef d'exploitation: C'est une variable quantitative qui représente l'âge ou le nombre d'années d'expérience du chef d'exploitation agricole. On s'attend ici à un impact de l'âge du chef d'exploitation sur l'adoption de la RNA. On suppose qu'un chef d'exploitation expérimenté est plus conscient des enjeux climatiques et environnementaux.

Niveau d'éducation du chef d'exploitation : Il s'agit d'une variable binaire qui prend la valeur 1 si le chef d'exploitation a fait l'école formelle et 0 si non. Cela pourrait affecter l'adoption de la RNA.

Le nombre d'actifs agricoles de l'exploitation: C'est une variable quantitative qui désigne le nombre d'actifs agricoles dans les exploitations. On présume que le nombre d'actifs agricoles est une variable importante qui affecte l'adoption des nouvelles technologies.

Superficie des exploitations : c'est une variable quantitative exprimée en hectare. On suppose que la possession des grandes superficies pourrait affecter la probabilité des 
exploitants à adopter ou rejeter les nouvelles technologies.

Tenure foncière: C'est une variable binaire qui prend la valeur 1 si l'exploitant est héritier et 0 si non. Le fait de posséder de parcelles par succession est un facteur qui influence le choix ou la prise de décision de l'exploitant sur le système de production de l'exploitation.

Fertilisants (organique ou chimique): L'utilisation des fertilisants est une variable binaire qui prend la valeur 1 si oui et 0 si non. On admet que l'utilisation des fertilisants dans les parcelles de culture est l'un des facteurs déterminants dans la productivité agricole et donc peut augmenter la probabilité des exploitants à adopter ou rejeter des technologies notamment la RNA.

Accès au crédit : Il s'agit d'une variable qui prend la valeur 1 si le chef d'exploitation a eu accès à un crédit quelconque et 0 si non. L'accès au crédit est un facteur qui permet de renforcer les moyens de production des exploitants agricoles.

Conseil/Contact avec les partenaires du développement agricole: C'est une variable qualitative qui prend la valeur 1 si l'exploitant a eu à collaborer avec l'un des partenaires au développement agricole. Il s'agit notamment $\mathrm{du}$ service de l'agriculture, la recherche agricole et les ONG qui assistent les exploitants en matière d'encadrement, d'appui financier et de diffusion des nouvelles technologies. Le fait de collaborer avec les partenaires du développement agricole affecte la probabilité d'adopter la RNA.

Le niveau de revenus : Il s'agit ici des revenus agricoles et extra-agricoles dont l'effet attendu pourrait être positif ou négatif sur l'adoption de la RNA.

Nombre d'unité bétail tropical (UBT): C'est une variable quantitative qui désigne le nombre d'unité bétail tropical dont dispose l'exploitation agricole. Le parcage des animaux dans les champs de culture et les parcelles dénudées favorisent le développement de la RNA et en contre- partie la RNA permet de satisfaire les besoins fourragers des animaux. En effet la possession d'animaux de parcage (bovins, ovins, caprins) peut influencer l'adoption de la RNA.

Cueillette: C'est une variable binaire qui prend la valeur 1 si l'exploitant pratique la cueillette et 0 si non. La cueillette permet en partie de pallier au problème d'insécurité alimentaire mais aussi elle contribue à l'amélioration des revenus du ménage et aux moyens de subsistance.

L'appartenance à une organisation ou association paysanne: Cette variable est qualitative et prend la valeur 1 si l'exploitant est membre d'un groupe et 0 sinon. L'appartenance à une association de type moderne (coopérative, groupement) ou traditionnelle (association de femmes, de jeunes) pourrait avoir une influence sur l'adoption de la RNA.

Perception sur la fertilité des parcelles : C'est une variable binaire qui prend la valeur 1 si bonne et 0 si non. Dans le souci d'améliorer la fertilité des parcelles de culture cette variable peut influencer de façon positive ou négative l'adoption de la RNA.

Perception sur le choc climatique: C'est une variable binaire qui prend la valeur 1 s'il y a eu choc climatique et 0 si non. Le choc climatique incite à prendre une décision sur l'adoption de la RNA.

Nous considérons que ces variables ont diverses caractéristiques. Il s'agit des caractéristiques socio-économiques des exploitants et exploitations agricoles (genre, âge, éducation, superficie, actifs agricoles, tenure foncière, fertilisants, cueillette, UBT, revenus agricoles, et extra-agricoles), des facteurs climatiques et environnementaux (fertilité des champs et le choc climatique), et des facteurs institutionnels (accès au crédit, conseil ou formation reçu à travers la recherche agricole, l'encadrement agricole et les ONG). 


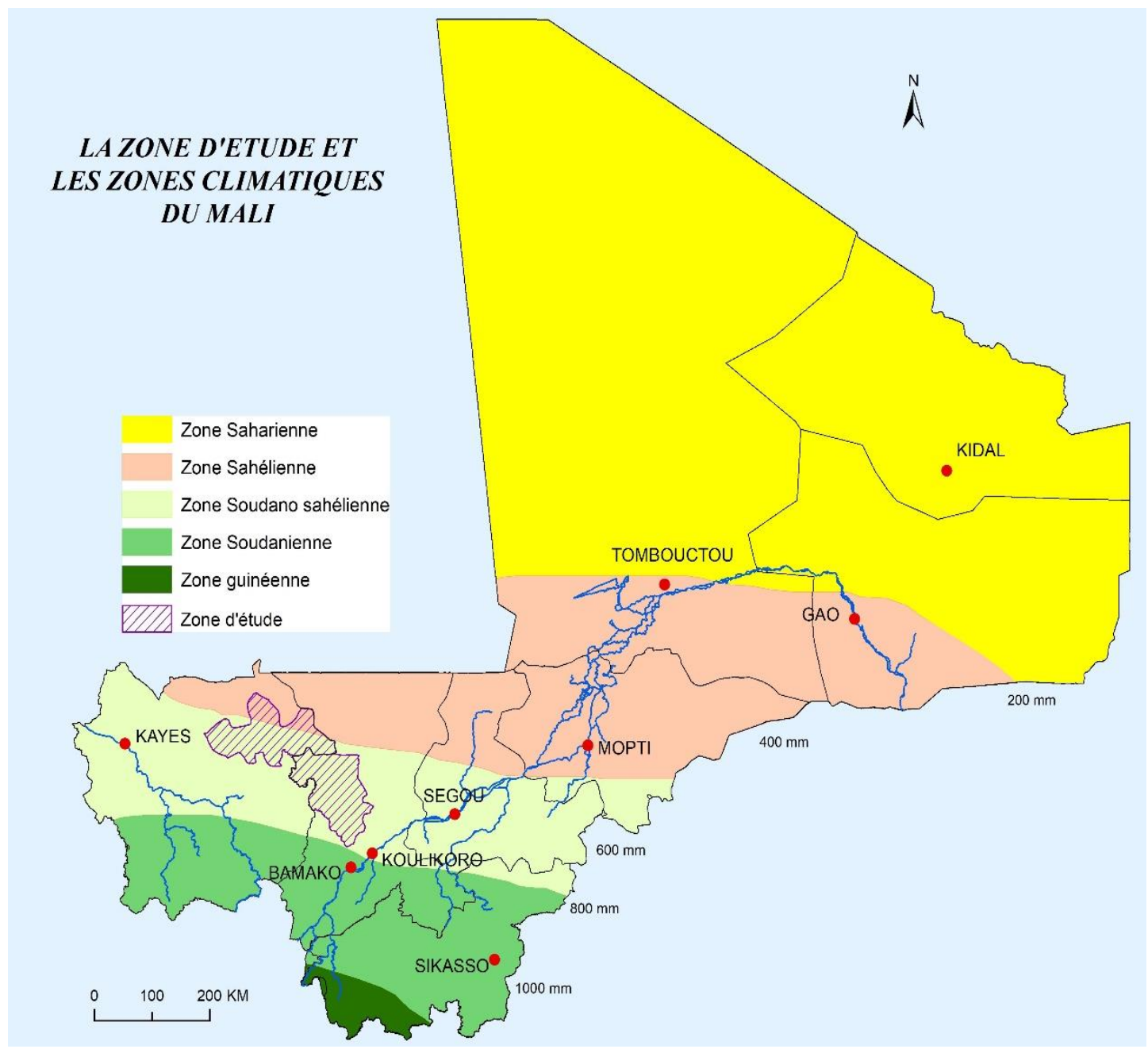

Figure 1: Localisation de la zone d'étude.

\section{RESULTATS}

\section{Caractéristiques socio-économiques des producteurs agricoles}

L'âge constitue un paramètre important dans l'adoption des technologies. Les résultats sont consignés dans le Tableau 1.

Ces résultats montrent que les chefs d'exploitation qui n'ont pas adopté sont relativement plus vieux que ceux qui ont adopté avec respectivement 57 et 52 ans. Il existe une différence significative au seuil de $1 \%$.

Dans l'ensemble, les résultats dans le Tableau 2 montrent un nombre important des producteurs chefs d'exploitation (soit $97,6 \%$ pour les adoptants contre $96,7 \%$ pour les non adoptants). Les femmes chefs d'exploitation sont peu nombreuses (environ 3\%). Cette situation s'explique par le faite que la fonction de chef d'exploitation est traditionnellement réservée aux hommes. Ces femmes sont dans la plupart des cas des veuves. Environ $97 \%$ des chefs d'exploitations sont mariés. La population de la zone d'étude est composée majoritairement de bambara $(68,3 \%$ pour les adoptants contre $66,7 \%$ pour les non adoptants). Les soninkés viennent en seconde position après les bambaras $(21,4 \%$ pour les adoptants et $30,4 \%$ pour les non adoptants). Les autres ethnies minoritaires sont les peulhs, 
les malinkés, les maures, les kagolo et les kassonkés. Les communautés de la zone d'étude pratiquent en majorité la religion musulmane $(86 \%$ pour les adoptants contre $82 \%$ pour les non adoptants). Les autres religions pratiquées sont l'animisme et le christianisme.

Le niveau de scolarisation des chefs d'exploitation est relativement faible, $35 \%$ (représentant la somme des taux des alphabétisés, du coranique, du primaire et du secondaire) des producteurs sont alphabétisés et $65 \%$ analphabètes. Ainsi, sur l'ensemble des chefs d'exploitation enquêtés, aucun n'a effectué des études supérieures. L'accès aux crédits agricoles est un facteur important pour renforcer les moyens de production des acteurs agricoles; en effet la plupart des producteurs affirment avoir accès au crédit soit $73 \%$ pour les non adoptants contre $65 \%$ pour les adoptants. Environ $78 \%$ des producteurs ont reçu des conseils auprès des services de développement agricole. Près de 79\% des producteurs adoptants ont bénéficié de formation sur les techniques de lutte anti érosive contre $68 \%$ pour les non adoptants. Ces formations portent essentiellement sur le compostage, les cordons pierreux, les fascines, les diguettes en terre et le zaï. En ce qui concerne la formation sur la RNA, environ $70 \%$ des producteurs adoptants affirment avoir été formé sur la RNA contre 15\% seulement pour les producteurs non adoptants (Tableau 2).

\section{Caractéristiques socioéconomiques des exploitations agricoles}

La population moyenne par exploitation agricole est constituée de 28 personnes pour les adoptants contre 29 pour les non adoptants (Tableau 3). La population active est de 10 personnes dont 5 actifs hommes et 5 actifs femmes soit un ratio moyen de 3 personnes à nourrir par actif agricole. Cette tendance est valable pour l'ensemble des exploitations.

Nous constatons également que l'exode rural est l'un des phénomènes le plus perceptible qui intéresse l'ensemble de la zone avec une moyenne de 4 personnes par exploitation agricole dont 3 hommes et une femme. Face aux handicaps naturels de la zone d'étude où vivent les populations démunies, l'exode apparaît dans bien des cas comme une solution aux difficultés rencontrées. Malgré qu'il détourne un grand nombre d'actifs, il assure néanmoins le complément nécessaire à la survie et à l'amélioration des conditions d'existence des populations.

Les résultats d'enquête montrent que les grandes superficies sont emblavées pour la culture du sorgho et du mil. La dominance des superficies du sorgho et du mil est liée à la forte contribution de leur production au besoin alimentaire des populations rurales. Les rendements obtenus par les producteurs adoptants sont estimés à $503 \mathrm{~kg} / \mathrm{ha}$ pour le sorgho, $528 \mathrm{~kg} / \mathrm{ha}$ pour le mil et $690 \mathrm{~kg} / \mathrm{ha}$ pour le maïs contre respectivement $481 \mathrm{~kg} / \mathrm{ha}, 458$ $\mathrm{kg} / \mathrm{ha}$ et $407 \mathrm{~kg} / \mathrm{ha}$ pour les producteurs non adoptants. En effet les rendements des cultures céréalières sont relativement plus élevés au niveau des exploitations adoptantes que celles non adoptantes mais en deçà de la moyenne nationale (Tableau 3).

\section{Analyse des déterminants de l'adoption de la régénération naturelle assistée (RNA)}

Les résultats issus de l'estimation du modèle Logit sont présentés dans le Tableau 4. L'analyse des résultats montre que le modèle est significatif. Les effets marginaux montrent l'impact de la variation d'une unité de chaque variable sur la probabilité d'adoption. L'analyse des résultats du modèle d'estimation des facteurs d'adoption de la régénération naturelle assistée montre que les variables, âge, formation en RNA et le revenu sont significatifs au seuil de $1 \%$; l'accès au crédit, la cueillette et l'unité bétail tropical sont significatifs au seuil de $5 \%$ et l'utilisation des fertilisants et la perception sur le choc climatique sont significatifs au seuil de $10 \%$. 
Tableau 1: Age moyen des chefs d'exploitations agricoles.

\begin{tabular}{llll}
\hline Variable & Adoptants & Non adoptants & P-value \\
\cline { 2 - 3 } & Moyenne & Moyenne & \\
\hline Age & $52,0 \pm 27,7$ & $57,4 \pm 26,1$ &, $000^{* * *}$ \\
\hline
\end{tabular}

Tableau 2: Caractéristiques des chefs d'exploitation agricoles.

\begin{tabular}{|c|c|c|c|c|}
\hline \multirow[t]{2}{*}{ Variables } & \multicolumn{2}{|c|}{ Adoptants $(\mathrm{n}=363)$} & \multicolumn{2}{|c|}{ Non adoptants $(n=231)$} \\
\hline & Effectif & $\%$ & Effectif & $\%$ \\
\hline \multicolumn{5}{|c|}{ Genre } \\
\hline Exploitant homme & 354 & 97,5 & 223 & 96,5 \\
\hline Exploitant femme & 9 & 2,5 & 8 & 3,5 \\
\hline \multicolumn{5}{|c|}{ Statut matrimonial } \\
\hline Marié & 354 & 97,6 & 223 & 96,6 \\
\hline Célibataire & 7 & 1,9 & 3 & 1,3 \\
\hline Veuf/veuve & 2 & 0,5 & 5 & 2,1 \\
\hline \multicolumn{5}{|c|}{ Ethnie } \\
\hline Bambara & 248 & 68,3 & 154 & 66,7 \\
\hline Soninké & 78 & 21,4 & 70 & 30,4 \\
\hline Peulh & 17 & 4,6 & 3 & 1,3 \\
\hline Maure, Kagolo, Malinké & 21 & 5,7 & 4 & 1,6 \\
\hline \multicolumn{5}{|c|}{ Religion } \\
\hline Islam & 314 & 86,4 & 189 & 81,9 \\
\hline Christianisme & 20 & 5,4 & 2 & 0,8 \\
\hline Animisme & 30 & 8,2 & 40 & 17,3 \\
\hline \multicolumn{5}{|c|}{ Niveau d'éducation } \\
\hline Analphabète (Non lettré) & 237 & 65,3 & 151 & 65,4 \\
\hline Alphabétisé & 51 & 14,1 & 28 & 12,2 \\
\hline Coranique & 16 & 4,4 & 18 & 8 \\
\hline Primaire & 51 & 14,1 & 29 & 12,7 \\
\hline Secondaire & 8 & 2,2 & 4 & 1,7 \\
\hline Accès au crédit & 236 & 65 & 169 & 73 \\
\hline Association/Groupement & 285 & 78,4 & 182 & 78,7 \\
\hline Conseil/contact & 315 & 86,8 & 160 & 69,3 \\
\hline Formation sur la LAE & 287 & 79,1 & 110 & 47,6 \\
\hline Formation sur la RNA & 254 & 70 & 35 & 15,2 \\
\hline
\end{tabular}

NB: $\%=$ pourcentage $; \mathrm{LAE}=$ lutte antiérosive 
Tableau 3: Caractéristiques socioéconomiques des exploitations agricoles.

\begin{tabular}{|c|c|c|c|}
\hline \multirow[t]{2}{*}{ Variables } & \multirow{2}{*}{$\begin{array}{l}\text { Adoptants } \\
\text { Moyenne }\end{array}$} & \multirow{2}{*}{$\begin{array}{l}\text { Non adoptants } \\
\text { Moyenne }\end{array}$} & \multirow[t]{2}{*}{ P-value } \\
\hline & & & \\
\hline Nombre d'hommes & $14 \pm 12,5$ & $14 \pm 12$ & 1,00 \\
\hline Nombre d'actifs-hommes & $6 \pm 4,9$ & $5 \pm 4,3$ & 0,33 \\
\hline Nombre d'hommes résidents & $11 \pm 9,5$ & $12 \pm 9,7$ & 0,52 \\
\hline Nombre d'hommes en exode & $3 \pm 4,3$ & $3 \pm 3,3$ & $0,09 *$ \\
\hline Nombre de femmes & $14 \pm 14,7$ & $15 \pm 15,4$ & 0,47 \\
\hline Nombre d'actifs-femmes & $5 \pm 5,8$ & $5 \pm 4,2$ & 0,33 \\
\hline Nombre de femmes résidentes & $13 \pm 13,9$ & $15 \pm 14,9$ & 0,4 \\
\hline Nombre de femmes en exode & $1 \pm 1,9$ & $0,4 \pm 1,4$ & $0,02 * *$ \\
\hline Taille de l'exploitation & $28 \pm 25,9$ & $29 \pm 26,2$ & 0,68 \\
\hline Actifs par exploitation & $11 \pm 9,9$ & $10 \pm 7,7$ & 0,29 \\
\hline Résidents par exploitation & $25 \pm 21,7$ & $26 \pm 23,5$ & 0,41 \\
\hline Exodes par exploitation & $4 \pm 5,3$ & $3 \pm 3,7$ & $0,02 * *$ \\
\hline Superficie du sorgho & $3,6 \pm 3,2$ & $3,5 \pm 2,2$ & 0,36 \\
\hline Superficie du mil & $4,7 \pm 4,3$ & $4,1 \pm 3,1$ & 0,83 \\
\hline Superficie du maïs & $1,5 \pm 1$ & $1,5 \pm 1,2$ & $0,00 * * *$ \\
\hline Production du sorgho & $2413,6 \pm 9734$ & $1669,6 \pm 1178,7$ & 0,26 \\
\hline Production du mil & $2519,1 \pm 2239,5$ & $1865,5 \pm 1415,9$ & 0,29 \\
\hline Production du maïs & $1061 \pm 813,8$ & $662,3 \pm 765$ & $0,00 * * *$ \\
\hline Rendement du sorgho & $503,3 \pm 87,1$ & $480,7 \pm 94,7$ & $0,06^{*}$ \\
\hline Rendement du mil & $528 \pm 84$ & $458,4 \pm 97,2$ & 0,72 \\
\hline Rendement du maïs & $689,5 \pm 222$ & $407 \pm 274,3$ & $0,00 * * *$ \\
\hline
\end{tabular}

NB : Significatif à $10 \%^{(*)} ;$ Significatif à $5 \%^{(* *)} ;$ Significatif à $1 \%$ (***) $^{(* *)}$ 
Tableau 4: Résultats de l'analyse des déterminants de la régénération naturelle assistée (RNA).

\begin{tabular}{|c|c|c|c|}
\hline Variables & $\mathbf{Z}$ & P-Value & Effets marginaux $(\mathrm{dy} / \mathrm{dx})$ \\
\hline Age & -2.67 & 0.008 & $-0.004 * * *$ \\
\hline Genre & 1.50 & 0.133 & 0.235 \\
\hline Niveau d'éducation & -0.60 & 0.551 & -0.030 \\
\hline Actifs agricoles & 0.37 & 0.709 & 0.001 \\
\hline Formation/RNA & 11.52 & 0.000 & $0.533 * * *$ \\
\hline Association villageoise & -0.45 & 0.652 & -0.024 \\
\hline Accès au crédit & -2.03 & 0.042 & $-0.101 * *$ \\
\hline Tenure foncière & -1.03 & 0.303 & $-0,097$ \\
\hline Superficie & -0.97 & 0.330 & -0.006 \\
\hline Fertilisants & 1.86 & 0.063 & $0.095^{*}$ \\
\hline Cueillette & 2.59 & 0.010 & $0.145^{* *}$ \\
\hline Revenus & 3.55 & 0.000 & $1.01 * * *$ \\
\hline UBT & -2.00 & 0.046 & $-0,005 * *$ \\
\hline Perception sur la fertilité des champs & 2.54 & 0.011 & $0.155^{* *}$ \\
\hline Choc climatique & -2.28 & 0.023 & $-0.245 * *$ \\
\hline \multicolumn{4}{|l|}{ Logistic regression } \\
\hline \multicolumn{4}{|l|}{ Number of obs $=594$} \\
\hline \multicolumn{4}{|l|}{ Prob $>$ chi $2=0.0000$} \\
\hline Pseudo R2 = 0.3441 & & & \\
\hline
\end{tabular}

\section{DISCUSSION}

\section{Influence de l'âge du chef d'exploitation sur l'adoption de la RNA}

L'âge du chef d'exploitation est une variable significative au seuil de $1 \%$ et influence de façon négative l'adoption de la RNA. Les personnes âgées sont moins susceptibles à adopter la RNA. Cela veut dire que les jeunes exploitants agricoles sont plus favorables à l'adoption de la RNA que les exploitants les plus âgées. Cela peut s'expliquer par le dynamisme et la curiosité des exploitants moins âgés dans l'adoption des nouvelles technologies. Ce résultat de l'influence de l'âge sur l'adoption est similaire à ceux obtenus par Issoufou et al. (2017). Il ressort de ces études que l'adoption de nouvelles technologies exige un certain niveau de risque associé à la décision du choix des technologies, les jeunes exploitants sont enclins à prendre plus de risque que les exploitants plus âgés. Par contre ce résultat sur l'influence de l'âge est contraire à ceux obtenus par Yabi et al. (2016) qui ont montré que les agriculteurs les plus âgés adopteraient plus facilement les innovations que les jeunes agriculteurs. Par ailleurs, une étude menée par Mounirou. (2015) dans le bassin cotonnier de Banikoara au Bénin a montré que la probabilité d'adoption n'est pas influencée par l'âge.

\section{Effet de la formation des exploitants sur l'adoption de la RNA}

Elle est l'un des principaux facteurs qui influence positivement l'adoption de la RNA dans la zone d'étude. Nous considérons que les exploitants formés sur la RNA disposent de plus d'informations sur les avantages et inconvénients de la technologie donc sont plus ouverts à son adoption. Ainsi la formation des exploitants entraine une augmentation de la probabilité d'adoption de la RNA. Ce résultat de la probabilité est soutenu par Zossou et al. (2009) qui ont trouvé qu'au Bénin, la 
vulgarisation des technologies agricoles par message vidéo est un facteur déterminant de la diffusion des technologies en milieu rural.

\section{Effet du niveau de revenus}

Nos résultats montrent que les revenus agricoles et extra agricoles augmentent la probabilité d'adoption de la RNA. Ces résultats confirment ceux obtenus par Mujinga et al. (2018) qui ont trouvé une relation positive entre le revenu et l'intention d'adoption de la mécanisation agricole. En effet ils affirment que plus l'agriculteur a un revenu agricole élevé plus il dispose de moyens de se procurer des nouvelles technologies et il utilise d'avantage les engrais minéraux afin de produire plus.

\section{Influence de la cueillette}

Malgré l'importance de la RNA dans l'approvisionnement des populations en produits de cueillette, des recherches scientifiques dans ce domaine sont peu documentés. La variable cueillette a une influence positive et significative sur l'adoption de la RNA au seuil de 5\%. Cela suppose que les exploitants qui pratiquent la cueillette des produits forestiers seraient plus intéressés sur l'adoption de la RNA afin d'augmenter leurs sources de revenus.

\section{Effet de l'utilisation des fertilisants}

L'utilisation des fertilisants est une variable significative au seuil de $10 \%$ et influence positivement l'adoption de la RNA. Ce résultat nous indique que les exploitants qui fertilisent leurs parcelles de cultures sont plus aptes à adopter la RNA en vue d'augmenter les rendements des cultures. Ce résultat corrobore aux résultats obtenus par Bello et al. (2012) qui ont trouvé que l'effet des doses d'engrais a été très hautement significatif sur la hauteur et la biomasse des mauvaises herbes ainsi que sur la hauteur des plants d'oignon à différentes périodes et le rendement en bulbes frais d'oignon.

Le résultat obtenu plus précisément sur l'application des fertilisants est contraire à celui de Mujinga et Afumba (2018) qui ont affirmé que l'utilisation des fertilisants ne semble pas jouer un rôle concernant l'intention d'adoption de la mécanisation agricole à Kimpese dans la province du Congo central. Il ressort également de nos résultats que les exploitants qui affirment avoir des champs fertiles ont un taux d'adoption de la RNA plus élevé que ceux ayant des champs moins fertiles. En effet l'utilisation des fertilisants ainsi que la perception sur la fertilité des champs augmenteraient respectivement l'intention d'adopter la RNA d'environ $10 \%$ et $16 \%$.

\section{Accès au crédit}

Dans la zone d'étude, la variable accès au crédit influence de façon négative l'adoption de la RNA au seuil de 5\%. Cela suppose que cette variable diminue la probabilité d'adopter la RNA de $10 \%$. Ce résultat est similaire à celui obtenu par Sodjinou et al. (2015) au Benin. Par contre les résultats des études réalisées par Fabrice et Yann. (2018); Sale et al. (2014) et Lawal et al. (2010) ont montré que l'accès au crédit est un facteur déterminant dans l'adoption de pratiques agricoles. Car, les producteurs ayant un accès facile aux crédits auront tendance à mieux adopter les nouvelles technologies.

\section{L’Unité Bétail Tropical (UBT)}

Les études socioéconomiques prenant en compte l'UBT comme variable sont très rares dans les publications. Cette variable est significative au seuil de 5\% et influence négativement l'adoption de la RNA. Ainsi les exploitants qui possèdent plus de bétail ont moins de chance d'adopter la RNA que ceux qui en possèdent peu. Plus la taille du cheptel est élevée, moins est la probabilité pour les paysans de pratiquer la RNA pour raison de besoin en fourrages pour les animaux. En disposant de peu d'animaux, les exploitants agricoles prouvent leur souci pour la protection de l'environnement en général et des ligneux en particulier.

\section{Perception sur le choc climatique}

Elle influence négativement la pratique de la RNA et est significative au seuil de 5\%. Cela explique que les exploitations touchées par le choc climatique adoptent moins la RNA que celles épargnées. Ce résultat corrobore 
celui obtenu par Mariano et al. (2012) qui ont estimé que les conditions climatiques comme les sécheresses répétées poussent les agriculteurs à rejeter certaines innovations pour des raisons techniques.

\section{Conclusion}

Les résultats de l'étude ont montré que les facteurs qui déterminent la probabilité d'adoption de la RNA sont d'ordre socioéconomique, environnemental et institutionnel. $\mathrm{Au}$ nombre de ceux-ci, la formation et le revenu sont les variables socio-économiques qui ont beaucoup influencées l'adoption de la RNA. Dans une optique de la promotion de la RNA en vue d'accroître son adoption et la résilience des exploitations agricoles face aux impacts des changements climatiques notamment dans les zones sahéliennes, nous suggérons un besoin accru en formation et la sensibilisation des agriculteurs et éleveurs sur les impacts socioéconomiques de la RNA.

\section{CONFLIT D'INTERETS}

Les auteurs déclarent qu'il n'y a pas de conflit d'intérêts.

\section{CONTRIBUTIONS DES AUTEURS}

DM a supervisé la collecte des données sur le terrain. Il a effectué les analyses statistiques des données collectées. Il a rédigé intégralement l'article. YK a lu l'article et a apporté des améliorations. KT a participé à la lecture de l'article. Il a proposé aussi des amendements pour l'amélioration de la qualité de l'article. ASM a lu l'article et a fait des suggestions afin d'améliorer son contenu. AMT a été le facilitateur du déblocage des fonds destinés aux travaux de collecte des données sur le terrain et à la publication du présent article.

\section{REMERCIEMENTS}

Cette étude a été réalisé grâce au financement du projet «Eco agriculture» de l'ONG World Vision. Nous tenons à remercier également les chefs d'exploitations agricoles pour les données ainsi que les lecteurs pour leurs observations.

\section{REFERENCES}

Agence de l'Environnement et du Développement Durable. 2017. Troisième Communication Nationale du Mali à la convention Cadre des Nations Unies sur les Changements Climatiques. Bamako, Mali. p.180. Available at http://aedd.gouv.ml/wpcontent/uploads/2018/08/RAPPORT_FI NAL_TCN_09_01_18.pdf

Bayala J, Sanou J, Teklehaimanot Z, Kalinganire A, Ouèdraogo SJ. 2014. Parklands for buffering climate risk and sustaining agricultural production in the Sahel of West Africa. Current Opinion in Environmental Sustainability, 6: 28-34. DOI: 10.1016/j.cosust.2013.10.004

Bello S, Ahanchede A, Amadji G, Gbehounou G, Aho N. 2012. Effet de la fumure minérale sur l'enherbement et la production de l'oignon (Allium cepa L.) au Nord-Est du Bénin. Int. J. Biol. Chem. Sci., $\quad$ 6(6): 4058-4070. http://ajol.info/index.php/ijbcs

Boko M, Niang I, Nyong A, Vogel C, Githeko A, Medany M, Osman-Elasha B, Tabo R, Yanda P. 2007. Climate Change 2007: impacts, adaptation and vulnerability. Contribution of Working Group II to the Fourth Assessment Report of the Intergovernmental Panel on Climate Change, M.L. Parry, O.F. Cambridge UK. pp. 433-467.

Fabrice KD, Yann ESM. 2018. Facteurs socioéconomiques influençant l'adoption de coton biologique au Nord- Est du Bénin: cas de la Commune de Kandi. International Journal of Progressive Sciences and Technologies (IJPSAT), 6(2) : 577-584. DOI : http://ijpsat.ijshtjournals.org.

FAO et ITPS, 2015. État des ressources en sols du monde - Résumé technique. Available at http://www.fao.org/3/a-i5126f.pdf. Rome, Italie.

FAO. 2016. Resilience Index Measurement Analysis II (RIMA-II). Available at http://www.fao.org/3/a-i5298e.pdf

Issoufou OH, Boubacar S, Adam T, Yamba B. 2017. Déterminants de l'adoption et impact des variétés améliorées sur la 
productivité du mil au Niger. African Crop Science Journal, 25(2) : 207 - 220. DOI :

http://dx.doi.org/10.4314/acsj.v25i2.6.

Lawal JO, Oduwole OO, Shittu TR, Muyiwa AA. 2010. Profitability of value addition to cashew farming households in Nigeria. Afr. Crop Sci. J., 19 (1): 49 - 54.

Mariano MJ, Villano R, Fleming E. 2012. Factors influencing farmers' adoption of modern rice technologies and good management practices in the Philippines. Agricultural Systems, 110(0): 41-53. DOI:

http://dx.doi.org/10.1016/j.agsy.2012.03. 010

Mortimore MJ, Adams WM. 2001. Farmer adaptation, change and 'crisis' in the Sahel. Global Environmental Change, 11(1): 49-57. DOI: 10.1016/S09593780(00)00044-3

Mounirou I. 2015. Perception et adoption des innovations techniques agricoles dans le bassin cotonnier de Banikoara au Bénin. African Journal of Agricultural and Resource Economics, 10(2): 87-102.

Mujinga K, Afumba N. 2018. Déterminants de la mécanisation agricole à kimpese dans la province du kongo central. Revue Congolaise d'Economie et de Gestion, EDUPC. pp.1-14. DOI : https://halamu.archives-ouvertes.fr/hal-01788077.

Nkonya E, Mirzabaev A, von Brau J. 2016. Economics of land Degradation and Improvement: a Global Assessment for Sustainable Development. Springer: New York; 215-260. DOI: http://dx.doi.org/10.1007/978-3-31919168-3

OCHA. 2014. Strategic Response Plan Sahel Region, United Nations Office for the Coordination of Humanitarian AEAA Sirs. Available on line at: https://docs.unocha.org/sites/dms/CAP/S RP_2014-2016_Sahel.pdf

Rosenstock TS, Lamanna C, Arslan A, Richards MB. 2015. What is the
Scientific Basis for Climate-smart Agriculture? CCAFS Info Note. Copenhagen, Denmark: CGIAR Research Program on Climate Change, Agriculture and Food Security. Available on line at: https://cgspace.cgiar.org/bitstream/handl e/10568/70258/http://InfoNote_CSA.pdf

Sale A, Folefack PD, Obwoyere GO, Lenah Wati N, Lendzemo WV, Wakponou A. 2014. Changements climatiques et déterminants d'adoption de la fumure organique dans la région semi-aride de Kibwezi au Kenya. Int. J. Biol. Chem. Sci., 8(2): 680-694. DOI: http://dx.doi.org/10.4314/ijbcs.v8i2.24

Sermé I, Outtara K, Logah V, Taounda JB, Pale S, Quansah C, Abaidoo R. 2015. Impact of tillage and fertility management options on selected soil physical properties and sorghum yield. Int. J. Biol. Chem. Sci. 9(3): 1154-1170.

DOI :

http://dx.doi.org/10.4314/ijbcs.v9i3.2

Sodjinou E, Glin LC, Nicolay G, Tovignan S, Hinvi J. 2015. Socioeconomic determinants of organic cotton adoption in Benin, West Africa. Agric. Food Econ., 3(12): $22 . \quad$ DOI: https://doi.org/10.1186/s40100-0150030-9

Yabi JA, Bachabi FX, Labiyi IA, Ode CA, Ayena RL. 2016. Déterminants socioéconomiques de l'adoption des pratiques culturales de gestion de la fertilité des sols utilisées dans la commune de Ouaké au Nord-Ouest du Bénin. Int. J. Bio. Chem. Sci., 10(2): 779-792.

DOI : http://dx.doi.org/10.4314/ijbcs.v10i2.27

Zossou E, Van Mele P, Vodouhe SD, Wanvoeke J. 2009. Comparing Video and Workshops to Train Rural Women about Improved Rice Parboiling in Central Benin. The Journal of Agricultural Education and Extension, 15(4): 329-340. DOI: 10.1080/13892240903309561. 